\title{
Entre traumatisme originel et choc fondateur : la Guerre Civile espagnole dans la série catalane Temps de silenci (TV3, 2001-2002)
}

\section{Jennifer Houdiard}

\section{(e) OpenEdition}

Journals

Édition électronique

URL : http://journals.openedition.org/tvseries/1858

DOI : 10.4000/tvseries.1858

ISSN : 2266-0909

Éditeur

GRIC - Groupe de recherche Identités et Cultures

Référence électronique

Jennifer Houdiard, «Entre traumatisme originel et choc fondateur : la Guerre Civile espagnole dans la série catalane Temps de silenci (TV3, 2001-2002) », TV/Series [En ligne], 10 | 2016, mis en ligne le 01 décembre 2016, consulté le 02 mai 2019. URL : http://journals.openedition.org/tvseries/1858 ; DOI : 10.4000/tvseries. 1858

Ce document a été généré automatiquement le 2 mai 2019.

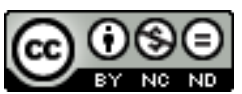

TV/Series est mis à disposition selon les termes de la licence Creative Commons Attribution - Pas d'Utilisation Commerciale - Pas de Modification 4.0 International. 


\title{
Entre traumatisme originel et choc fondateur : la Guerre Civile espagnole dans la série catalane Temps de silenci (TV3, 2001-2002)
}

\author{
Jennifer Houdiard
}

1 La chaîne de télévision publique catalane TV3 a diffusé ces dernières années de nombreuses fictions à caractère historique. Ces productions sont extrêmement variées : on peut citer par exemple La memòria dels Cargols (TV3/Dagoll Dagom, 1999), série humoristique relatant la vie d'une famille catalane du XIIIème siècle jusqu'à nos jours, ou Ermessenda (TV3, 2011), mini-série plus sérieuse consacrée à la comtesse Ermessende de Carcassonne, qui régna sur les comtés de Barcelone, Gérone et Osona au début du $\mathrm{XI}^{\mathrm{e}}$ siècle. Cette «mode » caractérise d'ailleurs, plus globalement, les séries produites dans l'ensemble de l'Etat espagnol depuis les années 2000, à tel point qu'un scénariste évoquait en 2011 dans une interview le risque que le public se lasse des séries historiques ${ }^{1}$.

2 Parmi les époques et événements abordés, la guerre civile et la dictature de Francisco Franco occupent une place de premier plan, comme dans un grand nombre de fictions contemporaines, qu'il s'agisse de la prose narrative ou du cinéma. La série espagnole Amar en tiempos revueltos (TVE, 2005-2012) -inspirée de la catalane Temps de silenci (TV3/ Diagonal TV, 2001-2002) dont il sera question ici - a connu un succès considérable. Plus récemment, une autre chaîne espagnole, Antena 3, a diffusé El tiempo entre costuras (Boomerang TV, 2013-2014), série que l'on pourrait qualifier de superproduction, avec son budget d'un demi-million d'euros par épisode ${ }^{2}$; elle a tiré sa révérence devant 5 millions de téléspectateurs le 13 janvier $2014^{3}$.

Bien que leurs formats et budgets soient très différents, Amar en tiempos revueltos et $\mathrm{El}$ tiempo entre costuras présentent un certain nombre de similitudes du point de vue de la diégèse : elles sont toutes deux centrées sur une jeune, belle et innocente protagoniste qui voit son existence -et notamment sa vie sentimentale- bouleversée par l'éclatement du conflit en 1936, puis par l'instauration en Espagne d'un régime dictatorial. Les 
circonstances historiques jouent un rôle déterminant du point de vue narratif, mais sont le plus souvent traitées de manière assez superficielle, voire grossière. Temps de silenci, fiction écrite par Rodolf Sirera, Gisela Pou et Enric Gomà et réalisée par Xavier Borrell, a été diffusée sur TV3 entre janvier 2001 et avril 2002. Comme la majorité des séries de fiction catalanes, elle a été produite conjointement par TV3 et la maison de production Diagonal TV.

4 Comme ses homologues espagnoles précédemment citées, Temps de silenci met sur le devant de la scène narrative une protagoniste féminine : il s'agit d'Isabel Dalmau (Cristina Dilla), fille d'un riche industriel barcelonais. Le téléspectateur découvre l'histoire d'Isabel depuis les mois précédant l'éclatement de la guerre civile espagnole, alors qu'elle a environ vingt ans, jusqu'à la fin du XXe siècle, moment du décès de la vieille dame qu'elle est devenue. La profusion de personnages secondaires permet de découvrir l'histoire d'autres familles, notamment celle de Ramon (Àlex Casanovas) avec qui Isabel vit un amour impossible pendant plus de trente ans. À travers la famille Comes, le monde ouvrier est donné à voir, tandis que les Dalmau représentent la bourgeoisie aisée. Le devenir de ces deux «clans» au fil des épisodes de la saga est intimement lié à la reconstitution de l'histoire mouvementée de l'Espagne et de la Catalogne, des années 1930 à l'aube du XXIème siècle.

5 Je tenterai de démontrer que le traitement de l'Histoire constitue un des traits d'originalité de Temps de silenci par rapport à ses homologues espagnoles : loin d'être un simple décor pour une romance tumultueuse, elle est bien souvent au premier plan, tandis que les amours contrariées des protagonistes se limitent généralement à une fonction d'illustration, voire de prétexte. A cet égard, l'évocation de la guerre civile espagnole dans cette série est particulièrement intéressante : le conflit qui sonne le glas de la relation naissante entre les protagonistes porte également un coup fatal à ce qu'était la Catalogne durant la Seconde République.

6 A travers l'itinéraire des personnages, protagonistes ou comparses, Temps de silenci raconte l'histoire de la Catalogne contemporaine comme celle d'une (re)construction après ce que l'on pourrait considérer comme un traumatisme originel ${ }^{4}$ : trois années de guerre, suivies de quatre décennies de répression ${ }^{5}$ visant à détruire la culture et l'identité catalanes, mais qui furent aussi quarante ans d'une résistance fertile et créatrice.

\section{Quand l'Histoire prend le pas sur la romance}

7 Le générique de Temps de silenci semble annoncer le début d'une fiction sentimentale; tout du moins, il en reprend les codes: le baiser passionné entre une infirmière et un soldat (figure 1), ainsi que la valse qui accompagne les images, constituent un seuil d'entrée dans la série relativement explicite. 
Figure 1 : le générique semble annoncer une fiction sentimentale

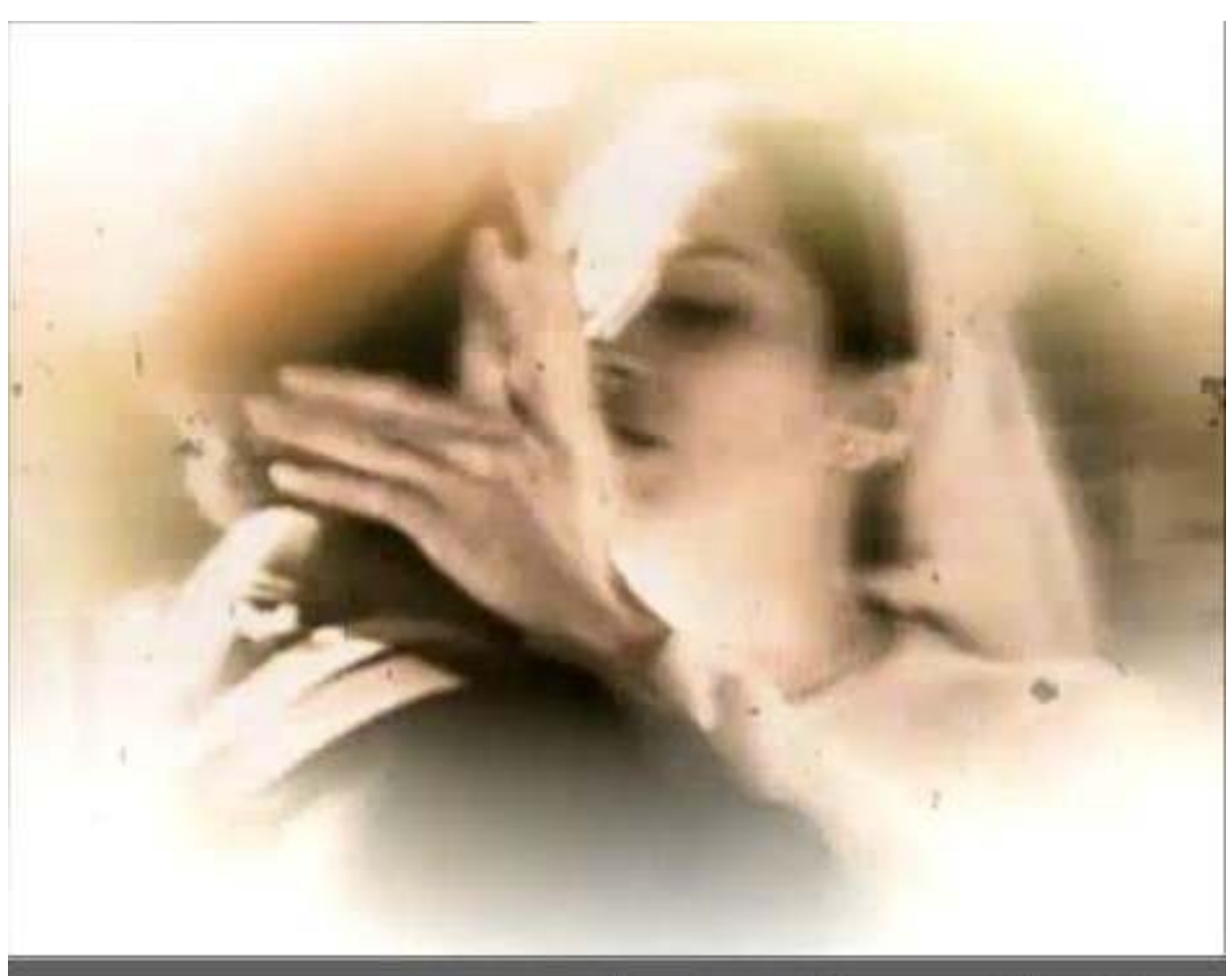

8 Dès la première séquence de l'épisode pilote, le ton est (apparemment) donné : la protagoniste, Isabel, évoque en voice over ses souvenirs de l'été 1935, tandis que les images montrent une famille bourgeoise de Barcelone se préparant à rejoindre sa résidence secondaire pour les vacances. Dernier été avant un bouleversement sans précédent, selon les mots de la narratrice : «L'amour ». Bouleversement d'autant plus important qu'Isabel est fiancée à un jeune homme de bonne famille et qu'elle a le mauvais goût de tomber amoureuse de Ramon, un ouvrier.

Cependant, le contexte politique de l'Espagne est très rapidement évoqué, d'abord à travers les informations radiophoniques qu'écoutent les personnages : il est fait mention $\mathrm{du}$ gouvernement Lerroux, récemment élu, et de ses difficultés. Les véritables bouleversements à venir commencent à se profiler, et c'est à leur rythme que l'intrigue se déroulera désormais. En effet, après la rencontre entre Isabel et Ramon, une première ellipse transporte le téléspectateur en janvier 1936, à l'aube des élections qui voient la victoire du Front Populaire. La montée de la tension dramatique repose, certes, sur la naissance d'une relation clandestine entre Isabel et le jeune ouvrier, mais aussi et surtout sur l'instabilité politique de la République espagnole dans les premiers mois de l'année 1936. La seconde ellipse importante prend d'ailleurs fin au mois de juillet, au moment du soulèvement des généraux insurgés: Isabel a rompu ses fiançailles et commence à fréquenter Ramon, au grand désespoir de sa famille. La relation entre les deux jeunes protagonistes est intimement liée par le récit aux événements politiques : sa temporalité leur est subordonnée, et ils la conditionnent à bien d'autres égards.

En effet, Temps de silenci évoque de manière précise et nuancée les conséquences de l'éclatement de la guerre civile en Catalogne, et notamment les mutations sociales qui en ont découlé. Contrairement au reste de l'Espagne, plutôt agricole, la Catalogne des années 1930 était une région fortement industrialisée, et se caractérisait par une bipolarisation 
de la société consciemment ressentie aux deux extrémités de l'échelle sociale ${ }^{6}$, ainsi que par une politisation importante de la classe ouvrière, au sein de laquelle les anarchistes étaient nombreux et très actifs. Au lendemain du 18 juillet 1936, la résistance face au fascisme s'organise, comme dans de nombreuses villes de l'État espagnol, à la différence près que les travailleurs prennent littéralement possession de Barcelone, à tel point que la bourgeoisie semble avoir disparu de l'espace public. L'historien Hugh Thomas affirme même que durant l'été 1936, il était mal vu de porter des vêtements ou accessoires suggérant l'appartenance aux classes aisées, et que les cravates et les chapeaux avaient pratiquement disparu des rues barcelonaises ${ }^{7}$. Cette ambiance de Grand Soir apparait clairement dans Temps de silenci : la guerre a éclaté, mais elle dynamite au passage l'ordre bourgeois et permet aux travailleurs de s'atteler à la construction d'un monde plus juste, de faire vivre l'utopie.

\section{Des personnages métonymiques}

11 On peut certes regretter une certaine simplification des événements historiques, notamment une représentation politiquement uniforme des républicains qui ne rend pas compte de la multiplicité des partis, courants et tendances ayant existé dans les faits ; de la même façon, les dissensions entre anarchistes, communistes du PSUC ${ }^{8}$ et du POUM ${ }^{9}$ et socialistes, ainsi que les violents affrontements qui ont divisé le camp républicain en mai 1937, sont passés sous silence. Tout se passe comme si la vérité historique était sacrifiée à la volonté de donner à voir l'union sacrée des travailleurs catalans face à l'oppression, qu'elle soit bourgeoise ou fasciste (l'apparente assimilation sera considérablement nuancée au fil de la diégèse, comme nous le verrons). On assiste à la réquisition de l'usine du père d'Isabel par ses ouvriers, tandis que, dans le salon de la famille bourgeoise, la radio annonce le vote d'un décret sur les collectivisations par la Generalitat, le gouvernement autonome catalan, comme pour souligner le rôle des institutions autonomiques dans la construction de cette nouvelle société durant les premiers mois de la guerre. C'est d'ailleurs ce contexte de "parenthèse enchantée ", caractérisé notamment par l'abolition, ou du moins l'atténuation, des inégalités de classes, qui donne à Ramon le courage de demander la main d'Isabel à sa mère (qui refuse). Le jeune couple finit par se marier en cachette -et en catastrophe- avant le départ de Ramon pour le front (figure 2). 
Figure 2 : Mariage d'Isabel et Ramon

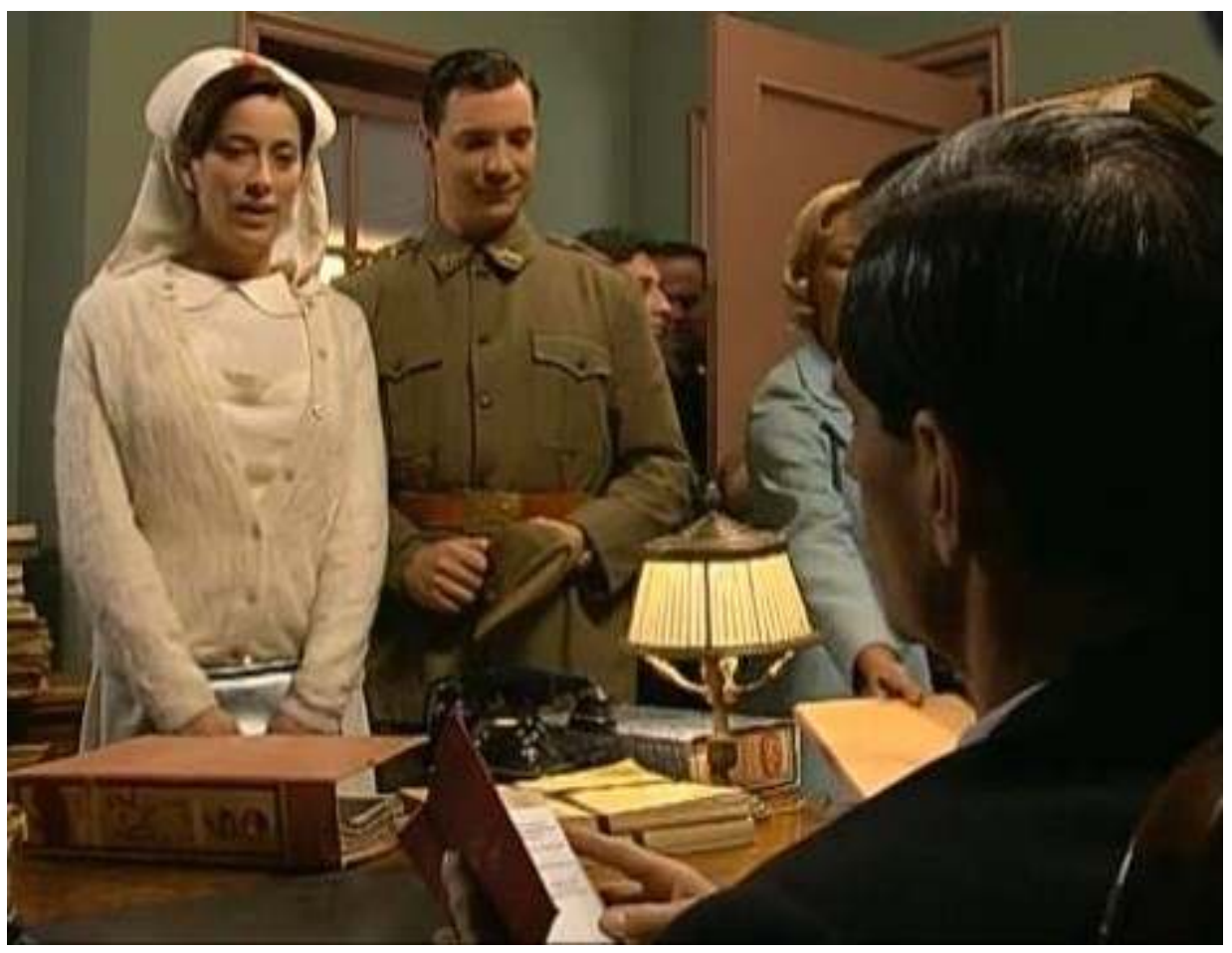

12 Au-delà de sa dimension dramatique, ce mariage est intéressant à plusieurs égards : en effet, il s'agit d'une union civile, qui ne sera pas reconnue par l'État franquiste après la guerre. L'intrigue principale de Temps de silenci est donc totalement conditionnée par les circonstances politiques et sociales : ce sont elles qui feront de la relation entre Isabel et Ramon un amour impossible. En effet, à la fin de la guerre, la nouvelle de la mort de Ramon décide Isabel à céder aux pressions familiales et à épouser Frederic (Pep Ferrer), un médecin ami de la famille. La non-validité de son précédent mariage rend cette seconde union possible, et d'autres facteurs étroitement liés au contexte politique et social la favorisent. Victòria (Isabel Rocatti), la mère d'Isabel, y joue un rôle déterminant : alors qu'elle sait que Ramon est vivant et emprisonné, elle choisit délibérément de garder le silence afin de pousser sa fille à contracter un mariage plus conforme à ses propres attentes. Le personnage de Victòria représente la bourgeoisie catalane: la victoire franquiste signifie pour elle la défense de ses intérêts de classe mis à mal durant la guerre (ce que symbolise l'union de sa fille avec un ouvrier).

13 De manière générale, de nombreux personnages de Temps de silenci apparaissent comme des archétypes, bien qu'il soit possible de nuancer et d'évoquer plutôt des personnages métonymiques, comme on le verra par la suite. Si l'on considère les amours malheureuses d'Isabel et Ramon comme l'intrigue principale, on peut dégager un schéma narratif relativement simple, dans lequel des figures caractérisées fort négativement jouent le rôle d'opposants. On peut penser à Victòria, la matriarche, une bourgeoise dure et sans scrupules qui fait passer le qu'en dira-t-on et l'intérêt de sa famille et de sa classe avant le bien-être et la liberté de ceux qui l'entourent. Elle est responsable du malheur de sa fille, et elle rejette son fils Llorenç (Pep Pla), qui a le tort de s'engager aux côtés des républicains pendant le conflit. En revanche, elle chérit plus que tout son autre fils, Quim (David Bagès), qui fait partie de la Phalange. On constate à travers l'opposition entre les deux frères la reprise, somme toute classique, du motif du conflit fratricide. 
La dichotomie est d'ailleurs poussée à l'extrême, car elle se situe sur deux plans, politique et axiologique. En effet, Quim apparait au début de la série comme un "méchant" exemplaire : il s'agit d'un jeune loup aux dents longues, prêt à prendre la direction de l'usine paternelle avec le projet d'être beaucoup moins conciliant que son père avec les ouvriers. Le fait que cette figure assez peu sympathique rejoigne le camp des insurgés dès les premiers jours du conflit laisse craindre un grossier manichéisme, et la représentation de la guerre civile comme un affrontement entre "mauvais franquistes» et «bons républicains ». Mais au fil des années, le personnage de Quim évolue : d'abord, quand le régime évacue peu à peu les phalangistes du gouvernement, il se sent trahi, d'autant qu'il a combattu sur le front russe avec la División Azul et qu'il en a gardé de lourdes séquelles physiques et psychologiques. Pendant les dernières années de la dictature, il finit par prendre ses distances avec le régime qu'il a contribué à amener au pouvoir. Cette rupture est symboliquement matérialisée par une tribune qu'il publie dans la presse contre la « justice militaire » suite au procès de Burgos (épisode 21).

La construction des personnages se révèle finalement assez fine et intéressante, et permet d'aborder des questions complexes. On constate rapidement que les « camps » ne sont pas si clairement délimités, surtout en Catalogne, où les enjeux sont multiples.

\section{La guerre civile ou la conquête coloniale de la Catalogne}

16 La victoire franquiste est clairement représentée comme une défaite dans Temps de silenci, à plus d'un titre. Défaite pour les républicains, bien sûr (soit la grande majorité des personnages caractérisés positivement dans la série), défaite aussi et surtout pour la Catalogne. D'abord parce que celle-ci est donnée à voir comme un bastion de la République, ensuite parce que la guerre civile a pris dans cette région une dimension de guerre coloniale. En effet, l'une des bases idéologiques du Movimiento Nacional ${ }^{10}$ était la défense de l'unité de l'Espagne autour d'une religion, d'une langue et d'une culture uniques. L'entrée de l'armée franquiste en Catalogne a été ressentie par beaucoup de combattants comme une conquête, celle d'un territoire « qui n'était pas l'Espagne et qu'il fallait espagnoliser ${ }^{11} »$. Dès l'entrée des troupes franquistes dans la ville de Lleida, en avril 1938, soit un an avant la fin de la guerre, Franco a aboli le Statut de Núria de 1932, qui conférait à la Catalogne une autonomie substantielle. Il s'agissait d'assimiler la Catalogne à l'Espagne, de gré ou de force, comme l'évoquent les historiens Josep Clara et Joan Puigbert :

La conquête de la Catalogne avait un sens beaucoup plus profond que la simple occupation d'un territoire plus ou moins important ou stratégique. C'était une conquête dont le but était d'établir une domination matérielle, mais aussi idéologique et culturelle dans un sens d'uniformisation, contraire aux principes différenciateurs reconnus par la République ${ }^{12}$.

17 L'une des manifestations les plus évidentes de cet esprit de conquête fut la persécution de la langue catalane dans l'espace public. Elle est amplement évoquée dans Temps de silenci, d'abord à travers un événement symbolique : la place barcelonaise sur laquelle vivent les personnages principaux est rebaptisée, son nom est castillanisé. Au début du quatrième épisode, la caméra montre la plaque "Plaça del blat ${ }^{13}$ ", dévissée et remplacée par une nouvelle plaque sur laquelle on peut lire «Plaza del trigo». 
Figure 3 : la place symboliquement rebaptisée

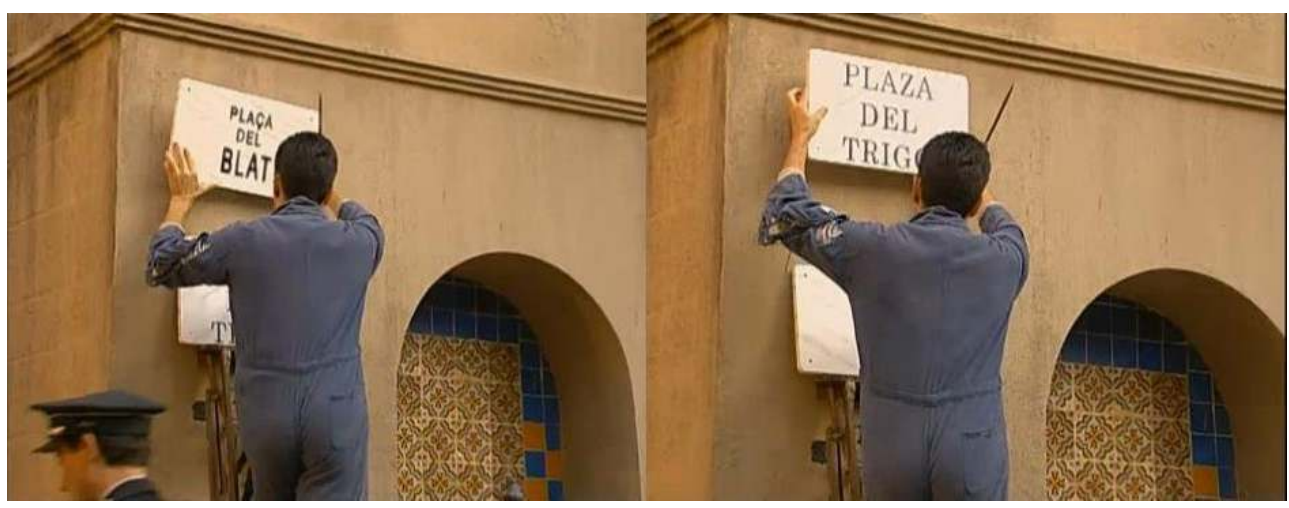

Cet épisode est situé chronologiquement dans les jours qui suivent la victoire de l'armée franquiste: ce plan annonce symboliquement les conséquences de l'instauration du nouveau régime sur la vie quotidienne des Catalans. Le climat répressif s'installe rapidement, ainsi que la peur et la soumission face à ceux qui apparaissent comme des occupants. Lorsqu'un soldat phalangiste fait irruption dans l'un des commerces du quartier, l'épicier s'empresse de changer de langue alors qu'il s'adressait en catalan à ses clientes. Quim, le frère d'Isabel, contraint les clients d'un café à se lever et à faire le salut fasciste quand la radio diffuse l'hymne national espagnol. Son beau-frère Frederic refuse d'obtempérer, et Quim le menace: "Maintenant, c'est nous qui faisons la loi ». Ce «nous" renvoie, en 1939, à un collectif uniforme, celui des vainqueurs de la guerre, auquel Quim revendique fièrement son appartenance. Il est persuadé d'avoir contribué à sauver l'Espagne, comme il le suggère lors d'une dispute avec Frederic lorsqu'il dit: « Nous avons apporté la paix ». Son beau-frère, catalaniste et républicain convaincu, lui répond: «Bien sûr. Une paix qui nous interdit le catalan, une paix qui impose son ordre et sa morale par la peur ».

\section{La résistance culturelle et linguistique catalane}

Le personnage de Frederic, le mari d'Isabel, est l'un des représentants de la résistance dans la série. A la différence des personnages de résistants politiques, majoritairement partisans de l'action et tôt ou tard victimes de la répression, il incarne une autre forme d'opposition plus rarement représentée dans les fictions. Il entreprend la rédaction d'une histoire de la médecine en Catalogne, et se nourrit de lectures prohibées par la censure franquiste, comme La nacionalitat catalana ${ }^{14}$ de Prat de la Riba, ouvrage considéré comme l'une des bibles du catalanisme politique. La figure de Frederic peut être lue comme une représentation de l'exil intérieur, à plus d'un titre: républicain, catalaniste et homosexuel, il mène une existence paisible de médecin, marié et père de famille, apparemment parfaitement intégré à une société dans laquelle il n'a en réalité pas sa place. Dans le onzième épisode de la série, alors qu'il évoque son désespoir face à la situation de la Catalogne dans l'Espagne franquiste, la révolte de Frederic trouve un écho inattendu : on entend une voisine chanter à tue-tête Els segadors, l'hymne catalan. Cette femme a perdu la raison après la mort de son fils, exécuté en raison de sa participation au conflit aux côtés des républicains. Comme Frederic, elle incarne une variation sur le thème de l'enfermement, réfugiée dans sa folie, mais malgré tout symboliquement 
rebelle, à travers cet hymne interdit qui résonne dans tout l'immeuble comme un défi face à l'oppression.

La résistance apparaît également à travers les pratiques culturelles et artistiques de certains personnages: en 1961, quelques habitants du quartier montent une troupe de théâtre amateur, sous la direction d'un ancien comédien. Celui-ci choisit de représenter $\mathrm{El}$ Café de la Marina ${ }^{15}$, une pièce de Josep Maria de Sagarra, figure de la vie littéraire clandestine dans la Catalogne de l'après-guerre. La fable relate l'histoire d'un amour secret, et fait écho à la situation d'Isabel et Ramon : plus de dix ans après leur mariage et leur séparation forcée, ils vivent dans le même quartier, sur la même place, avec leurs conjoints et enfants respectifs, et sont contraints de dissimuler leurs sentiments encore intacts. Bien évidemment, un concours de circonstances fait que ce sont justement les anciens amants qui se retrouvent face à face sur scène pour incarner les protagonistes d'El Cafè de la Marina.

21 Ainsi, Isabel et Ramon peuvent se déclarer leur amour au grand jour, le temps de la représentation, à travers les personnages qu'ils interprètent. Au-delà de sa fonction de métaphore de l'histoire d'amour malheureuse des protagonistes, cet épisode a un autre intérêt: il permet d'évoquer le rôle fondamental de l'art et de la culture dans la sauvegarde de l'identité catalane. En effet, si la dictature a porté un coup fatal au théâtre commercial catalan, le théâtre amateur s'est développé dans les années 1950. C'est dans ce contexte qu'a été fondée en 1955 l'Agrupació Dramàtica de Catalunya, dont l'objectif était de développer un théâtre national de qualité, de promouvoir les œuvres d'auteurs catalans et de traduire les textes des grands dramaturges étrangers. Ainsi, la pratique d'amateurs comme les personnages de Temps de silenci reflète une effervescence créatrice qui a permis la naissance du théâtre indépendant catalan, aujourd'hui reconnu comme l'un des plus dynamiques et novateurs au monde, dans un contexte pourtant peu propice. Soit dit en passant, l'épisode est explicitement daté dans la série, et cette date mérite commentaire : 1961 est la date de création d'Òmnium Cultural, société civile dont l'objectif était (et est toujours) de favoriser le développement de la culture catalane ${ }^{16}$. Òmnium Cultural a notamment soutenu financièrement l'Agrupació Dramàtica de Catalunya ${ }^{17}$, ainsi que l'Institut d'Estudis Catalans ${ }^{18}$.

Il importe par ailleurs de préciser que les personnages de Temps de silenci ne délaissent à aucun moment leur langue maternelle, malgré son strict cantonnement à la sphère privée. Tout au long de la série, dont les derniers épisodes sont chronologiquement situés au début du XXIe siècle, le catalan reste la seule langue parlée par les personnages, à l'exception des figures d'immigrés venus du Sud de l'État espagnol à partir des années 1950. Certes, la réalité a été beaucoup plus complexe, mais le fait est que Temps de silenci donne à voir une véritable résistance linguistique qui monte en puissance à mesure que les années passent. Il s'agit d'abord d'une résistance passive : le castillan n'est parlé par les personnages catalanophones que lorsque le contexte l'exige, face aux forces de l'ordre, par exemple. L'évolution des personnages de migrants andalous est intéressante à cet égard: Paco (Miquel Gelabert), qui s'installe dans l'immeuble d'Isabel dans les années 1950, commence rapidement à essayer de parler le catalan, et ses voisins l'encouragent vivement. Son fils Antonio (Julio Manrique), qui a pourtant grandi en Andalousie, parle presque exclusivement catalan : comme de nombreux personnages d'enfants d'immigrés dans les fictions catalanes ${ }^{19}$, il incarne un modèle d'intégration réussie.

23 La défense active de la langue apparaît de manière plus discrète dans la série: les revendications et leur évolution au fil du temps sont matérialisées par des plans réguliers 
sur des graffitis. Dans les années 1940, les murs sont constellés d'inscriptions anticatalanistes ("Si eres español, habla español ${ }^{20}$ ", épisode 4). Peu à peu, les graffitis changent de camp, et les revendications catalanistes s'expriment: dans un épisode situé au début des années 70 , on peut lire une inscription exigeant l'enseignement du catalan à l'école (épisode 21, voir figure 4).

Figure 4 : « Du catalan à l'école ! »

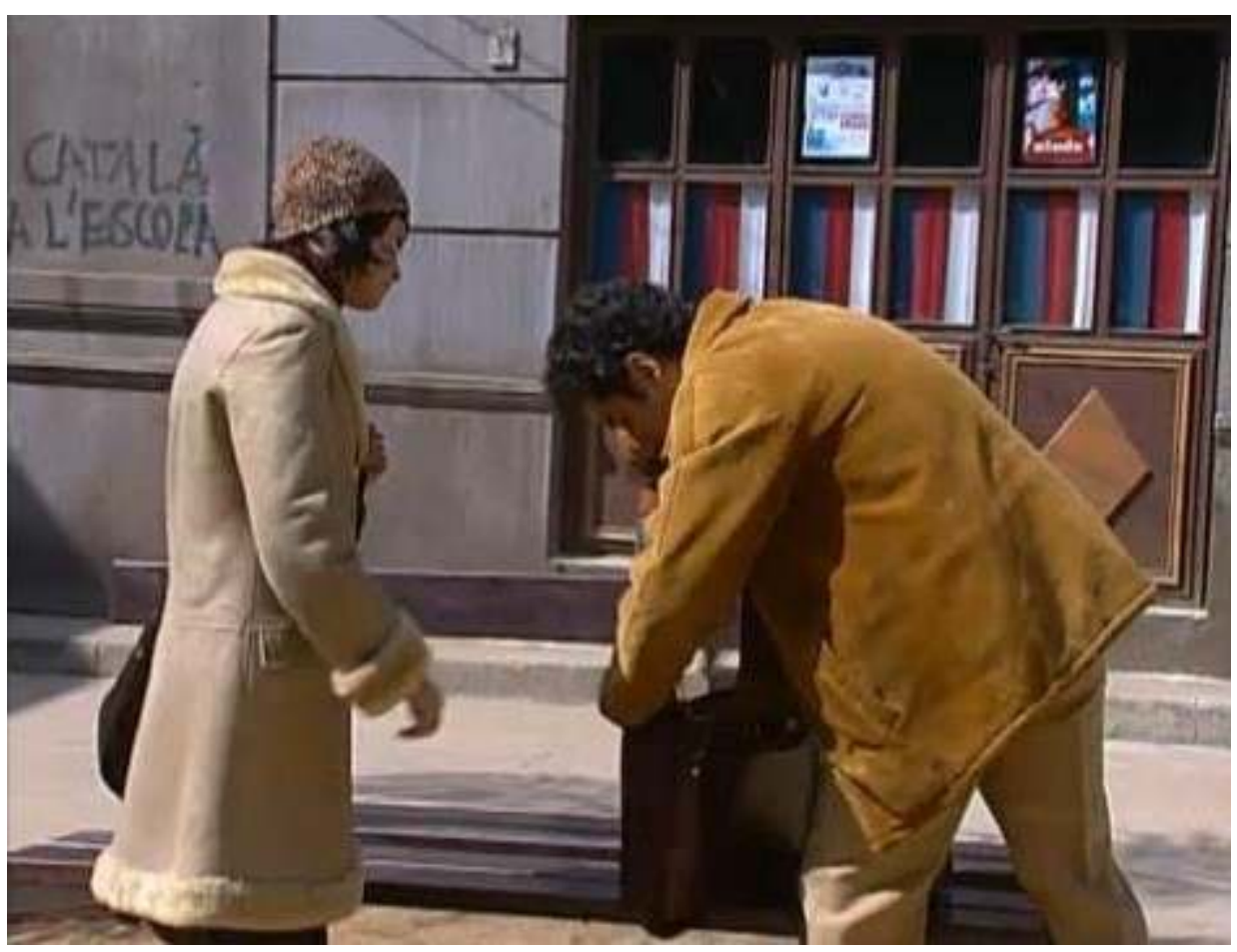

L'un de ces graffitis semble particulièrement intéressant : une main anonyme réclame au coin d'une rue des évêques catalans («Volem bisbes catalans », épisode 20, voir figure 5). On peut y voir une évocation discrète de l'apparition et du développement d'un nouveau catalanisme, bourgeois et catholique, parallèlement au catalanisme républicain de gauche ${ }^{21}$. À partir des années 1950, les revendications émanent de différents secteurs de la population, et la résistance se généralise: Temps de silenci évoque plus ou moins explicitement cette montée en puissance et cette diversification de l'engagement pour la défense de la culture et de la langue catalane. 
Figure 5 : « Nous voulons des évêques catalans »

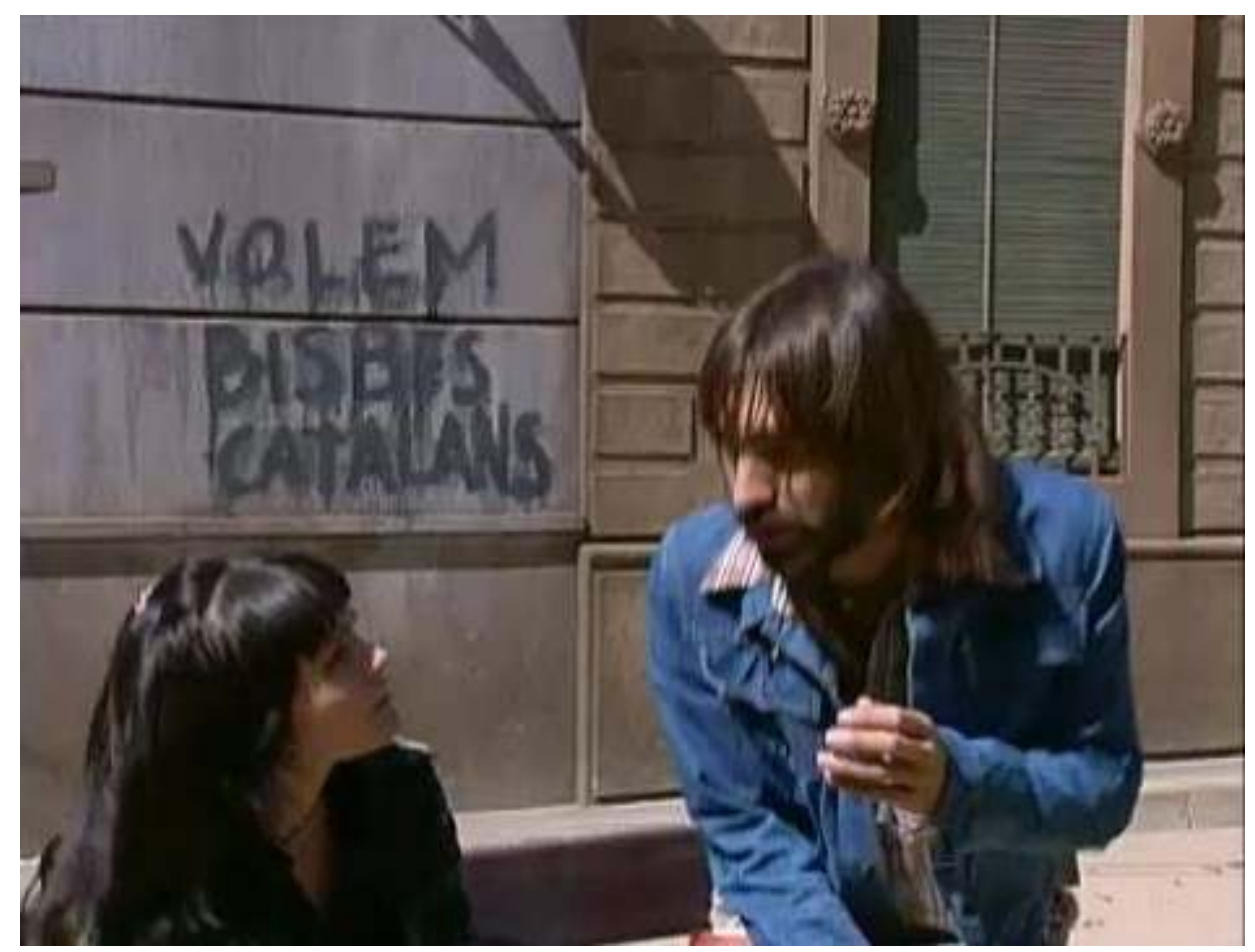

A travers l'histoire d'Isabel et de Ramon et de leurs entourages respectifs, c'est une page de l'histoire de la Catalogne qui est donnée à lire. Leurs amours contrariées apparaissent comme le fil conducteur de la diégèse, sur lequel se greffe une structure qui apparaît comme une série de cercles concentriques, selon Charo Lacalle, spécialiste des fictions télévisées catalanes :

Cette structure narrative s'articule en trois cercles concentriques: la trame sentimentale, qui tient lieu de fil conducteur de l'histoire ; les événements politiques et sociaux, dont la fonction est de doter chaque chapitre d'un référent, et les petits événements quotidiens touchant les personnages, qui servent essentiellement à récupérer la mémoire sociale collective de l'époque remémorée. Le résultat est un récit de fiction qui offre une reconstruction amène et didactique de la mémoire historique: la vraisemblance de ce récit est due à l'articulation pertinente des différents éléments qui le composent ${ }^{22}$.

La romance centrale peut également être lue comme une sorte de métaphore de la résistance au franquisme, car son évolution est parallèle à celle de la situation politique $\mathrm{du}$ pays. Durant la dictature, Isabel et Ramon sont contraints de dissimuler leurs sentiments, mais ne cessent jamais de s'aimer. En 1973, Isabel se décide à quitter son mari pour Ramon, qui est veuf, au moment où la mort du président du gouvernement Carrero Blanco porte un coup fatal à un régime déjà affaibli. Ramon est candidat aux premières élections démocratiques, en 1977, et meurt le lendemain du scrutin. Isabel lui survit quelques temps, jusqu'au jour où la Catalogne rend hommage à Ramon : son nom est donné à une place de son village d'origine. Le plan sur le dévoilement de la plaque fait écho à l'épisode de la place barcelonaise débaptisée au début de la dictature franquiste, et se donne à lire comme une revanche, d'autant qu'elle est recouverte par la senyera, le drapeau catalan (voir figure 6). 


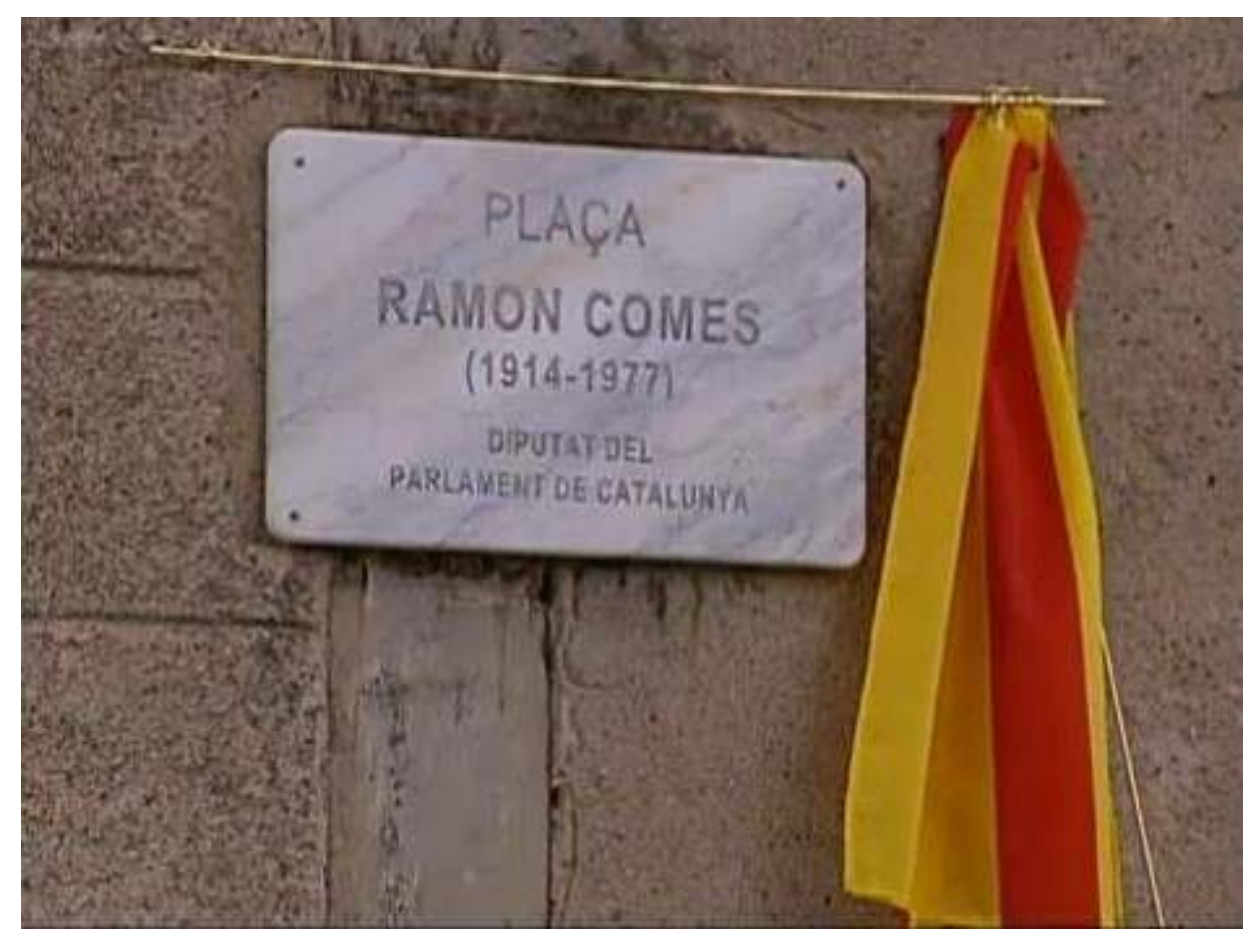

Dans un reportage télévisé consacré à Ramon, la commentatrice évoque son engagement comme « un travail silencieux et constant de résistance au régime qui a rendu possible la Transition démocratique actuelle dans notre pays » (épisode 40). Le soir de la diffusion du reportage, le présentateur annonce que toutes les télévisions de l'Etat espagnol doivent rendre l'antenne. Nous sommes le 23 février 1981, une tentative de coup d'État est en train de se dérouler; les personnages craignent que la démocratie n'ait été qu'une parenthèse et que les militaires ne reprennent le pouvoir. Une fois la situation maîtrisée, ce n'est pas le célèbre discours du roi Juan Carlos que la famille d'Isabel écoute à la radio, mais celui de Jordi Pujol, président de la Generalitat de Catalunya : symboliquement, c'est une voix catalane qui rassure le peuple quant à la continuité de la jeune démocratie. Les droits des Espagnols, mais aussi et surtout des Catalans, sont désormais garantis. C'est à la fin de cet épisode qu'Isabel s'éteint : le couple protagoniste n'a plus de rôle, ni politique ni narratif, à jouer dans l'histoire, ni dans l'Histoire. L'héroïne disparaît, passant le relais à sa fille Núria, avocate et députée communiste, future mère célibataire, qui assumera désormais le rôle de personnage central et de narratrice et mènera d'autres combats.

\section{Conclusion : la reconstruction de l'identité catalane}

Temps de silenci donne à voir l'histoire d'une nation construite dans l'adversité: la

Catalogne autonome de la Seconde République, victime d'une impitoyable répression, a été patiemment rebâtie, à travers différentes résistances, différents engagements, dans tous les milieux sociaux. C'est cependant un "pays» différent qui a été construit et continue d'être construit par les personnages de la série, des années 1940 à l'aube du XXI siècle : la guerre et la dictature font partie de son histoire et de sa mémoire, de la même façon que les différentes luttes menées par les Catalans pour conserver leur identité et leurs droits mis à mal durant le franquisme. Cela fait écho à un autre choc fondateur, 
celui de la chute de Barcelone durant la guerre de Succession d'Espagne, en 1714 : fait original et symbolique, c'est une défaite lourde de conséquences ${ }^{23}$ que commémorent les Catalans chaque 11 septembre. La loi du 12 juin 1980, qui a proclamé officiellement la Diada comme fête nationale catalane, rappelle le lien entre adversité et résistance qui a fait de la Catalogne ce qu'elle est aujourd'hui :

La reconstruction nationale des peuples passe, sans aucun doute, par la récupération de leurs institutions de gouvernement propres. Elle passe également par la valorisation et l'exaltation de tous les symboles à travers lesquels les communautés s'identifient, car ces symboles sont une synthèse de tous les facteurs historiques, sociaux et culturels qui constituent les racines de toute réalité nationale. Parmi ces symboles se distingue l'existence d'un jour de fête pendant lequel la Nation exalte ses valeurs, se remémore son histoire et les hommes qui en furent les protagonistes, et se projette dans l'avenir. Au cours de ses années de lutte, le peuple catalan a choisi le 11 septembre pour célébrer sa fête nationale. Date qui commémore le douloureux souvenir de la perte des libertés, le 11 septembre 1714, ainsi qu'une résistance active face à l'oppression, mais qui signifie aussi l'espoir en la reconstruction nationale. Aujourd'hui, alors que la Catalogne reprend le chemin de la liberté, les représentants du peuple croient que la Chambre Législative doit acter ce que la Nation a décidé à l'unanimité24.

\section{BIBLIOGRAPHIE}

"Guionistes avisen que el públic se saturarà de sèries històriques", Regio7.cat, 10/06/2011, http:// www.regio7.cat/televisio/2011/06/10/guionistes-avisen-que-public-se-saturara-serieshistoriques/148825.html, consulté le 02/06/2014.

« Siete días entre costuras », El Mundo, 14/01/2014, http://www.elmundo.es/ television/2014/01/14/52d56e8422601d497c8b4573.html, consulté le 02/06/2014.

AGUADO Elsa, « 'El tiempo entre costuras' o la joya de la corona de Antena 3 : todas las claves”, Vertele.com, http://www.vertele.com/video-articulo/el-tiempo-entre-costuras-o-la-joya-de-lacorona-de-antena-3-todas-las-claves/, consulté le 02/06/2014.

CLARA Josep et Joan Puigbert, « Els darrers mesos de la Catalunya autónoma », in Catalunya i la guerra civil, éd. Jaume Sobrequés i Callicó, Barcelone, Edicions d'Ara, 1983, p. 443-493.

DE RIQUER Borja et Joan B. Culla, « El franquisme i la transició democràtica (1939-1988) », éd.

Pierre Vilar, Història de Catalunya, Barcelone, Edicions 62, 2000, p. 305.

DUBoIS Jacques, Les romanciers du réel, Paris, Seuil, 2000.

GUTIÉRREZ Y MARTíNEZ Jesús María, « Del 18 de juliol als fets de maig de 1937 », in Catalunya i la guerra civil, éd. Jaume Sobrequés i Callicó, Barcelone, Edicions d'Ara, 1983, p. 24 [p. 7-280].

LACALLE Charo, « Èxits i fracassos. Anàlisi de cas : Temps de silenci i Cuéntame cómo pasó », La producció de ficció televisiva a Espanya, Quaderns del CAC, Barcelone, Consell de l'Audiovisual de Catalunya, novembre 2002, p. 37-46. 
PONS PRADES Eduardo, Los niños republicanos en la guerra de España, Madrid, Oberón, 1997.

PRAT DE LA RIBA Enric, La nacionalitat catalana, Barcelone, Edicions 62, 1978 [1906].

SAGARRA Josep Maria, El Cafè de la Marina, Barcelone, Millà, 1933.

THOMAS Hugh, La guerre d'Espagne, Tome 1, Paris, Robert Laffont, 1961.

VILLAROYA Joan « Des de l'etabliment del Govern de la República a Catalunya fins a la batalla de l'Ebre », Catalunya i la guerra civil, éd. Jaume Sobrequés i Callicó, Barcelone, Edicions d'Ara, 1983, p. 323-440.

\section{NOTES}

1. "Guionistes avisen que el públic se saturarà de sèries històriques", Regio7.cat, 10/06/2011, http://www.regio7.cat/televisio/2011/06/10/guionistes-avisen-que-public-se-saturara-serieshistoriques/148825.html, consulté le 02/06/2014.

2. Elsa Aguado, «'El tiempo entre costuras' o la joya de la corona de Antena 3 : todas las claves”, Vertele.com, http://www.vertele.com/video-articulo/el-tiempo-entre-costuras-o-la-joya-de-lacorona-de-antena-3-todas-las-claves/, consulté le 02/06/2014.

3. "Siete días entre costuras", El Mundo, 14/01/2014, http://www.elmundo.es/ television/2014/01/14/52d56e8422601d497c8b4573.html, consulté le 02/06/2014.

4. Jacques Dubois parle de «traumatisme originel » à propos de la Révolution française lorsqu'il évoque l'histoire mouvementée du XIXe siècle. Cf. Les romanciers du réel, Paris, Seuil, 2000, p. 150.

5. L'offensive des adversaires de la République pendant la Guerre Civile, ainsi que la répression qui a suivi le conflit, ont été particulièrement violentes en Catalogne, car ses habitants étaient presque automatiquement considérés comme des dissidents en puissance : «Voici ce qu'a déclaré un officier franquiste à Santa Liña, début avril 1938 : «Le simple fait d'être catalans fait de vous des Rouges et justifie qu'on vous exécute ». Cela répondant sans doute à ce que proposait le Diario de Burgos : « Nous devons agir en Catalogne comme en territoire conquis [...] ». Plus clair encore : à un journaliste qui l'interrogeait au sujet du "problème catalan", un officier franquiste répondit, catégorique : «Le problème catalan ? Il est facile à résoudre : il suffit de tuer tous les catalans ! C'est juste une question de temps ». [...] Un historien catalan, Solé Sabaté, nous offre un délicieux florilège de citations «historiques". Deux échantillons : "Chiens catalans! Vous n'êtes même pas dignes du soleil qui vous éclaire ", « La Catalogne devrait être semée de sel », Eduardo Pons Prades, Los niños republicanos en la guerra de España, Madrid, Oberón, 1997., p. 194. Je traduis.

6. Jesús María Gutiérrez y Martínez, «Del 18 de juliol als fets de maig de 1937 », éd. Jaume Sobrequés i Callicó, Catalunya i la guerra civil, Barcelone, Edicions d'Ara, 1983, p. 24 [p. 7-280].

7. Hugh Thomas, La guerre d'Espagne, Tome 1, Paris, Robert Laffont, 1961, p. 269.

8. Partit Socialista Unificat de Catalunya, parti catalan d'idéologie marxiste-léniniste.

9. Partido Obrero de Unificación Marxista, parti espagnol marxiste hétérodoxe qui s'est notamment distingué par son opposition au stalinisme.

10. Appareil d'État de l'Espagne franquiste de 1937 à 1976.

11. Joan Villaroya, « Des de l'etabliment del Govern de la República a Catalunya fins a la batalla de l'Ebre ", éd. Jaume Sobrequés i Callicó, Catalunya i la guerra civil, op. cit., p. 346 [p. 323-440]. Je traduis.

12. Josep Clara, Joan Puigbert, «Els darrers mesos de la Catalunya autónoma », ibid., p. 444 [p. 443-493]. Je traduis.

13. «Place du blé ».

14. Enric Prat de la Riba, La nacionalitat catalana, Barcelone, Edicions 62, 1978 [1906].

15. Josep Maria de Sagarra, El Cafè de la Marina, Barcelone, Millà, 1933. 
16. Borja de Riquer, Joan B. Culla, El franquisme i la transició democràtica (1939-1988), éd. Pierre Vilar, Història de Catalunya, Barcelone, Edicions 62, 2000, p. 305.

17. Ibid., p. 306.

18. Institution dont les missions sont comparables à celles de structures telles que l'Institut de France.

19. Pensons au personnage de Rafa dans Ventdelplà (TV3, 2005-2010) ou à celui de Cruz dans Infidels (TV3/Diagonal TV, 2009-2011).

20. «Si tu es espagnol, parle espagnol ».

21. Pensons par exemple au collectif C.C. (qui peut renvoyer à «Crist i Catalunya » ou à « Catòlics Catalans»), né en 1954, qui rassemblait des catholiques issus des mouvements scouts et de diverses associations religieuses. Cf Borja de Riquer, Joan B. Culla, El franquisme i la transició democràtica (1939-1988), op. cit., p. 300-301.

22. Charo Lacalle, "Èxits i fracassos. Anàlisi de cas: Temps de silenci i Cuéntame cómo pasó ", La producció de ficció televisiva a Espanya, Quaderns del CAC, Barcelone, Consell de l'Audiovisual de Catalunya, novembre 2002, p. 45 [p. 37-46].

23. En effet, suite à la chute de Barcelone après un siège de plus d'un an, les Catalans ont payé cher leur résistance face à Philippe V. Outre les destructions causées par les combats (un tiers de la ville détruite et des milliers de morts), ils subirent une répression féroce, notamment à travers les Decret de Nova Planta de 1716, qui mirent fin à l'indépendance de la Couronne d'Aragon (dont faisait partie la Principauté de Catalogne), supprimèrent les institutions officielles catalanes et interdirent l'usage du catalan dans l'administration publique.

24. Llei 1/1980, de 12 de juny, per la qual es declara Festa Nacional de Catalunya la diada de l'onze de setembre, http://www20.gencat.cat/portal/site/portaljuridic/ menuitem.d15a4e5dfb99396dc366ec10b0c0e1a0/?action=fitxa\&documentId=1732, consulté le 07/06/2014. Je traduis.

\section{RÉSUMÉS}

Depuis quelques années, les fictions à caractère historique se multiplient sur la chaîne publique catalane TV3. De La memòria dels Cargols (TV3, 1999), série humoristique relatant la vie d'une famille catalane durant sept siècles, à Ermessenda (TV3, 2011), mini-série centrée sur Ermessende de Carcassonne, qui régna sur les comtés de Barcelone, Gérone et Osona au début du XI ${ }^{\mathrm{e}}$ siècle, ces productions ont en commun de faire découvrir aux téléspectateurs quelques pages de l'histoire de cette «Nation sans État » qu'est la Catalogne, et d'explorer ainsi les origines et la construction d'une identité particulière, forgée dans l'adversité et maintes fois mise à mal.

La série Temps de silenci (TV3, 2001-2002) constitue un exemple intéressant de ce traitement fictionnel de l'Histoire: elle raconte les amours contrariées d'Isabel Dalmau, fille d'un riche industriel barcelonais, et de Ramon Comes, employé dans l'usine de ce dernier, depuis les derniers mois de la Seconde République espagnole jusqu'à la Transition démocratique, en passant par les longues années de dictature du général Franco. L'un des traits d'originalité majeurs de cette série, si on la compare à des productions du même type, comme la série espagnole Amar en tiempos revueltos (TVE, 2005-2012), est le traitement de l'Histoire : au lieu d'être une toile de fond un peu grossière, un simple décor pour une romance tumultueuse, elle est bien souvent au premier plan dans Temps de silenci. A cet égard, l'évocation de la guerre civile espagnole dans 
cette série est particulièrement intéressante : le conflit qui sonne le glas de la relation naissante entre Isabel et Ramon porte également un coup fatal à ce qu'était la Catalogne durant la République. A travers l'itinéraire des personnages, protagonistes ou comparses, Temps de silenci raconte l'histoire de la Catalogne contemporaine comme celle d'une (re)construction après ce que l'on pourrait considérer comme un traumatisme originel : quatre décennies de répression visant à détruire la culture et l'identité catalanes, mais aussi et surtout quarante ans de résistance pour que continuent à vivre une langue et une culture, autant d'années de lutte dont les Catalans d'aujourd'hui sont les héritiers.

The Catalan public TV channel TV3 broadcasted many historical fictions recently, such as La memòria dels Cargols (TV3, 1999), a humoristic show which tells the history of a Catalan family over seven centuries, or Ermessenda (TV3, 2011), a miniserie about Ermessinde of Carcassonne, Countess consort of Barcelona, Girona and Osona in the $11^{\text {th }}$ century. All these productions offer the audience the opportunity of discovering the history of Catalonia, a "stateless nation", and explore the origins and the construction of a peculiar identity which had to build itself in spite of (or thanks to) many misfortunes and threats.

The TV show Temps de silenci deals with history in a very interesting and original way: it tells the frustrated love story between Isabel Dalmau, a wealthy Barcelonese industrial's daughter, and Ramon Comes, one of her father's employees, from the last months of the Spanish Second Republic to the Democratic Transition, and during Francisco Franco's dictatorship. One of the most original features of this show is the way it deals with history, which is far from being a simple, rough setting for a romance, as in shows such as the Spanish Amar en tiempos revueltos (TVE, 2005-2012). In Temps de silence, the history of Catalonia appears to be the main character, and the evocation of the Spanish Civil War is worth an analysis: the conflict puts an end to Isabel and Ramon's relationship as well as to what Catalonia had become during the Second Republic. Through the characters' destiny, Temps de silenci tells the history of Catalonia along the 20th century as if it was the story of a (re)construction after a sort of original trauma: four decades of repression which aimed at destroying the Catalan culture and identity but appeared to be as well four decades of resistance to defend a language and a culture, a struggle inherited by today's Catalans.

\section{INDEX}

Keywords : Spain, Catalonia, Spanish Civil War, resistance, identity

Mots-clés : Espagne, Catalogne, guerre civile espagnole, résistance, identité

\section{AUTEUR}

\section{JENNIFER HOUDIARD}

Jennifer Houdiard est maître de conférences à l'université de Nantes, où elle enseigne la littérature espagnole contemporaine, la traduction et la communication. Elle a consacré sa thèse de doctorat à l'étude de la construction des personnages féminins dans les romans de Rosa Regàs. Ses recherches actuelles portent sur la prose narrative espagnole et catalane contemporaines, sur les adaptations cinématographiques de romans, ainsi que sur la télévision catalane, avec un intérêt particulier pour la représentation de l'identité dans la fiction (identité nationale, particulièrement en Catalogne, mais aussi et surtout identité de genre). Jennifer Houdiard is an Associate Professor in Contemporary Spanish Literature at the University of Nantes, where she also teaches Translation and Communication. In her PhD she studied the 
construction of female characters in Rosa Regàs' novels. She works on Spanish and Catalan literature, cinema (especially film adaptations) and TV series, and her research focuses on representations of gender and national identities in the Spanish and Catalan contemporary fiction. 